\title{
Correction: Cannabis use and driving-related performance in young recreational users: a within-subject randomized clinical trial
}

In an article published Oct. 14, 2018, ${ }^{1}$ Lynda Cedar was meant to have been acknowledged. The acknowledgement statement has been updated at cmajopen.ca.

\section{Reference}

1. Ogourtsova T, Kalaba M, Gelinas I, et al. Cannabis use and driving-related performance in young recreational users: a within-subject randomized clinical trial. CMA7 Open 2018;6:E453-62.

CMAJ Open 2020. DOI:10.9778/cmajo.20200040 